\title{
ARTIGO
}

dO https://doi.org/10.22481/praxisedu.v16i40.6903

\section{LA EMPATÍA EN ESTUDIANTES UNIVERSITARIAS DE EDUCACIÓN PRIMARIA}

\author{
EMPATHY IN PRIMARY SCHOOL UNIVERSITY STUDENTS
}

EMPATIA EM ESTUDANTES UNIVERSITÁRIOS DE ESCOLAS PRIMÁRIAS

\author{
Ana María Carty-Vásquez \\ Universidad César Vallejo, Lima - Perú
}

Janet Josco-Mendoza

Universidad Continental, Lima - Perú

Yolvi Ocaña-Fernández

Universidad Privada San Juan Bautista, Lima - Perú

Resumo: $\mathrm{O}$ artigo teve como objetivo determinar a dimensão predominante da variável empatia, bem como a dimensão e o indicador predominantes, respectivamente; Para isso, o Teste do Índice de Reatividade Interpessoal para 139 estudantes do Ensino Fundamental da Universidade de Ciências e Humanidades e da Universidade Nacional de San Marcos. A pesquisa apresentou a abordagem quantitativa do tipo substantivo básico. O desenho descritivo não-experimental de predominância foi selecionado. Os resultados indicaram que não há predominância de qualquer dimensão ou indicador porque quase todos os participantes eram mulheres com treinamento consciente em habilidades sociais. Foi ratificado as fontes referenciadas no campo da pedagogia, uma vez que existe uma estreita relação entre as duas dimensões da empatia como parte da formação humanística integral dos futuros professores.

Palavras-chave: Empatia, Cognitiva, Afetiva.

\begin{abstract}
Article aimed to determine the predominant dimension of the empathy variable as well as the predominant dimension and indicator respectively; For this, the Interpersonal Reactivity Index (IRI) for 139 Primary Education students of the University of Science and Humanities and the National University of San Marcos. The research presented the quantitative approach of the basic substantive type. The non-experimental descriptive descriptive design of predominance was selected. The results indicated that there is no predominance of any dimension or of any indicator because almost all the participants were women with conscious training in social skills. Was ratified the sources referenced in the field of pedagogy since there is a close relationship between both dimensions of empathy as part of the integral humanistic formation of future teachers.
\end{abstract}

Keywords: Empathy, Cognitive, Affective. 
Resumen: El artículo tuvo como objetivo determinar la dimensión predominante de la variable empatía así como la dimensión y el indicador predominantes respectivamente; para ello, se utilizó el Test de Índice de Reactividad Interpersonal (IRI) en 139 estudiantes de Educación Primaria de la Universidad de Ciencias y Humanidades y de la Universidad Nacional Mayor de San Marcos. La investigación presentó el enfoque cuantitativo del tipo básico sustantivo. Se seleccionó el diseño no experimental descriptivo explicativo de predominancia. Los resultados indicaron que no hay predominancia de ninguna dimensión ni de ningún indicador porque casi todos los participantes fueron mujeres con formación consciente en competencias sociales. Se ratificó las fuentes referenciadas en el campo de la pedagogía puesto que hay una relación estrecha entre ambas dimensiones de la empatía como parte de la formación humanística integral de los futuros profesores.

Palabras clave: Empatía, Cognitivo, Afectivo.

\section{Introduction}

Empatía es un término que se repite mucho y aparece en numerosos estudios; pero estos pertenecen, en su mayoría, al campo de la psicología o la medicina; no obstante, su presencia en el campo educativo es casi nula; lo anterior genera una preocupación puesto que la empatía favorece el desarrollo de habilidades blandas como trabajar en equipo y ser un buen líder; por ende, todo estudiante durante su formación profesional requiere el conocimiento de la empatía a partir de uno de sus aspectos: cognitivo, perceptivo, comportamental o emocional. A lo largo del tiempo, la empatía se ha definido como un afecto compartido, una reacción emocional, una respuesta afectiva vicaria; es decir, sentir lo que la otra persona siente.

La Organización de las Naciones Unidas para la Educación, la Ciencia y la Cultura (UNESCO 2015) publicó una primera guía "Educación para la ciudadanía mundial: Temas y objetivos de aprendizaje" con el fin de que los ciudadanos se sientan pertinentes a la humanidad internacional mediante la educación. La guía supone tres dimensiones básicas: 1) cognitivo, 2) socioemocional y 3) conductual. El constructo empatía se ubica en la dimensión socioemocional. Los resultados informan que es necesario el aprendizaje de actitudes empáticas por los estudiantes de educación superior con una mirada crítica para que sean solidarios y respeten las diferencias. También la UNESCO (2017) publicó una guía "Soporte socioemocional. Reconstruir sin ladrillos” en la cual define empatía como la capacidad de ponerse en el lugar de otra persona en una situación determinada y comprender cómo la 
persona se está sintiendo. El propósito de esta guía fue repotenciar las capacidades de los estudiantes de gestionar sus emociones.

Por otro lado, Bautista, Vera, Tánori y Valdés (2016) señalaron la ausencia de instrumentos para medir la empatía en México y en Latinoamérica, gran parte de instrumentos provienen de Europa y EE. UU; por ello, los estudios latinoamericanos emplean versiones de estos en lengua castellana.

A través de los medios nacionales de comunicación, se observa mucha intolerancia y violencia debido a la falta de empatía; por lo expuesto, el papel de la educación superior es fundamental. La Ley General de la educación peruana, 28044, (2012) en sus artículos 2, 8 y 9 , expresó su inquietud por la formación plena de los educandos y que la educación debe promover valores y actitudes positivos que permitan una convivencia adecuada a partir de la enseñanza- aprendizaje del manejo de las emociones.

La Ley N 28740 (2006) o Ley del Sistema Nacional de Evaluación, Acreditación y Certificación de la Calidad Educativa (Sineace) definió y estableció criterios que deben cumplir las instituciones para garantizar la calidad de su enseñanza con el fin de que los universitarios adquieran competencias que garanticen su permanencia en el mercado laboral. Una de estas capacidades es la empatía. De lo expuesto, Sevillano (2016) señaló que la acreditación responde a un perfeccionamiento continuo de la calidad de la enseñanza en la instrucción superior, una calidad que no solo debe ser exclusiva de los europeos; sino de los latinoamericanos; por ello, esta búsqueda constante de perfectibilidad, de corrección, de toma de decisiones idóneas empezó en Chile y recién se observó en nuestro país a partir de los años 90.

En cuanto a la universidad peruana, específicamente en el campo de la educación, los estudios sobre la empatía son escasos; de allí la importancia de conocer las conductas empáticas de los discentes de educación superior para, a partir de los resultados que se obtengan, poder repotenciarlas. La gran mayoría de investigaciones se ha centrado en estudiantes médicos, residentes, sicólogos; pero no en alumnos de Educación Primaria, quienes serán futuros maestros que formarán niños y necesitan informarse sobre cómo se sienten los demás para poder brindar una enseñanza de calidad. Por todo lo anterior, se investigó la empatía en estudiantes de Educación Primaria de dos universidades ya que son muy pocos los estudios descriptivos explicativos de la empatía en alumnos de dos centros de educación superior. Este estudio sería uno de los primeros a nivel nacional en enfocar la empatía en estudiantes de Educación. Por lo expuesto, surgió el problema general de 
investigación que fue ¿Cuál es la dimensión predominante de la empatía en estudiantes de los últimos ciclos de Educación Primaria de dos universidades peruanas?

De acuerdo a la realidad mencionada, se consideró antecedentes que dieran solidez a la investigación. En el plano internacional, se halló muchas investigaciones enriquecedoras como la de Ulloque, Villalba, Varela, Fantini, Quinteros, Díaz (2019), en Argentina, quienes mostraron diferencias significativas en el nivel de empatía por año escolar dado que los alumnos de quinto empatizaban más que los de primero; del mismo modo, el nivel de empatía iba aumentando conforme pasaban los años académicos; la otra diferencia sustancial fue el nivel de empatía por género pues las mujeres empatizan más que los varones.

En España, Giménez, Prado y Valero (2019) midieron la comunicación, la empatía y la inteligencia emocional. Los resultados mostraron puntajes significativos de acuerdo al tipo de contrato laboral en el que se encontraban las participantes: permanente, temporal e interino; por ejemplo, cuando hay una seguridad en el puesto de trabajo, o sea, las de contrato permanente, estas habilidades puntúan significativamente; por lo tanto, son mejores las relaciones empáticas entre enfermeras y pacientes. También en España, Maldonado y Barajas (2018) efectuaron una investigación para ver la relación entre la empatía y la teoría de la mente. Los resultados indicaron que los niños tienen una mejor empatía afectiva; es decir, compartir experiencias emocionales; por otro lado, la habilidad de tomar conciencia sobre los sentimientos de las demás personas va aumentando conforme pasan los años.

Alves, Rodrigues y Ribeiro (2018), en Brasil, investigaron la influencia de los sentimientos empáticos en la conducta altruista de adultos. Los resultados demostraron que las personas tienden a empatizar y ayudar a otros siempre que no arriesguen demasiado y el costo a pagar no sea demasiado alto. También en Brasil, Arango, Olivera, Restrepo y Puerta (2018) realizaron una evaluación multidimensional de la empatía. Los autores comprobaron que la empatía es la mejor forma de reducir las conductas agresivas y antisociales puesto que la dimensión cognitiva que implica una respuesta empática a la angustia del otro o leer estados emocionales mostró un puntaje muy favorecedor.

Moreira, DeSouza y Guerra (2018) así como Merino, Sánchez y Revilla (2018) y Zabala, Richards, Breccia y López (2018) aplicaron el instrumento Interpersonal Reactivity Index (IRI). Los autores concluyeron que los resultados de empatía fueron buenos, sobre todo en las mujeres que empatizaron más que los varones; pero los datos obtenidos deberían ser mejores puesto que se trata de futuros maestros que deben ser empáticos para poder transmitir 
esta capacidad; por ende, manifestaron la necesidad de que haya más estudios que midan la empatía de los próximos educadores.

Guzmán (2018), en Costa Rica, realizó un estudio sobre comunicación empática entre educadores y estudiantes tanto dentro como fuera de las aulas. Los datos obtenidos mostraron que la empatía permite el diálogo y la estabilidad emocional de los niños puesto que se sienten importantes; de igual forma, un constante balance de la empatía en la práctica docente es parte de la responsabilidad social de cada maestro. En Ecuador, Dávila, Neira, Aguilera, Martínez, Vélez y Días (2017) encontraron diferencias significativas en los niveles de empatía según el nivel académico (el año que cursan) y el género pues las mujeres mostraron más empatía (mayor sensibilidad emocional) que los hombres dado que las decisiones de estos tienden a ser más racionales. En Bolivia, Pérez (2019) reflexionó sobre la formación humana en valores de los futuros profesionales; entre ellos, el de la empatía. Los resultados obtenidos, luego de aplicar distintos talleres y sesiones, demostraron la necesidad de incluir la formación ética en carreras con alta responsabilidad social puesto que se tratará con pacientes.

En Colombia, Fortich y Díaz (2018) describieron el grado de actitud empática según género y niveles de enseñanza. Se concluyó que la orientación empática iba aumentando conforme se pasaba de un año a otro. También en Colombia, Pulido y Herrera (2018) midieron las variables felicidad e inteligencia emocional. Los resultados encontraron una relación proporcional entre las dos variables. La edad, las condiciones de vida negativa, la cultura y el género fueron muy influyentes en el factor empatía que se encontró dentro de la variable inteligencia emocional. También en Colombia, López, Arias, González y García (2018) midieron el grado de relación entre la empatía en sus dos dimensiones (afectiva y cognitiva). Los resultados demostraron que la edad fue significativa en ambas variables puesto que influyó tanto en los procesos creativos así como en la capacidad de colocarse en el sitio de alguien más; es decir, se evidenció de forma positiva en la dimensión de empatía afectiva.

En el plano nacional, se halló muchas investigaciones sobre la empatía aplicadas en realidades diferentes, que en su mayoría se asocian a las facultades de Psicología y Medicina, con instrumentos variados como es el caso de Palomino y Almenara (2019), quienes evidenciaron que las mujeres tienen un mayor nivel de empatía debido a factores sociales, educacionales y personales. Similares resultados obtuvieron Huarcaya y Encinas (2018) quienes indicaron que los niveles de empatía aumentaron en estudiantes mujeres. Del mismo modo, Corrales (2017) indicó que hay un nivel moderado de empatía en más del $50 \%$ de 
participantes y que la dimensión predominante fue la empatía cognitiva; esto coincide exitosamente con trabajos anteriores.

En cambio, Mayo, Quijano, Ponce y Ticse (2019) demostraron que no hay diferencias significativas ni de género ni de estado civil entre los participantes como otras investigaciones han encontrado; por otro lado, los autores concluyeron que el nivel de empatía aumentó en aquellos que llevaron programas complementarios, entrenamientos o talleres de empatía. Por otro lado, Concha, Ccarita, Marroquín y Atamari (2018) probaron que la empatía fue un poco más significativos en los varones; por lo tanto, no siempre las mujeres empatizan mejor por lo que el nivel de empatía no se debe solamente al sexo; sino a otros factores como experiencias personales o el estado emocional que presentaron los estudiantes al momento de responder los ítems.

Luego de revisar diversas investigaciones, enfoques e instrumentos utilizados, se concluyó que son muchos los factores que se deben considerar al momento de medir el nivel de empatía en los participantes. Los autores examinados coincidieron en que la empatía debe ser considerada dentro de la formación profesional de cualquier persona y reforzada mediante talleres. Una comunicación empática entre maestros, alumnos, pacientes, etc. reducirá los diferentes tipos de violencia que se presentan a diario en todas partes de un mundo globalizado y; por ende, impersonal o deshumanizado debido, justamente, a la poca falta de empatía.

Los estudios sobre empatía nunca serán suficientes porque aportan en reducir los problemas de índole social; más bien, la falta de información sobre el tema sería atentar contra el proceso de rehumanización que urge en el mundo entero. En el último año, se observó un incremento de violencia tanto fuera como dentro del hogar y los niños se están formando dentro de familias observando conductas agresivas y asimilándolas; por consiguiente, la necesidad de conocer el nivel de empatía que tienen los futuros docentes que se encargarán de guiar y formar a los niños

Se consideró como punto de partida del marco teórico a Altuna (2018) quien revisó diversas explicaciones para concluir que estamos viviendo la "era empática" como una de las principales prácticas morales, que parte de un nivel básico (transferencia de emociones), luego uno asociativo (con una experiencia propia parecida) hasta la adopción de la perspectiva imaginativa (ponerse en el lugar de otro real o ficticio); este último es el nivel más complejo que puede darse centrándose en uno mismo, centrándose en el otro o combinando ambas perspectivas. 
De igual modo, Arenas y Jaramillo (2017) señalaron que el término empatía no presenta una sola definición ni siquiera en el campo sicológico. Los autores desarrollaron un recorrido histórico interdisciplinario de la palabra desde el filósofo Adam Smith, en 1759, quien la conceptualizó como "sentir pena por el dolor del otro"; Tichener, en 1909, quien la tradujo del inglés al alemán que significa "compenetrarse"; Kohler, en 1929, quien fue el pionero en la investigación de la empatía cognitiva y la entendió como comprender los sentimientos de las otras personas; en el campo sicoanalítico, Freud, año 1921, la conceptualizó como una transferencia de emociones entre médico y paciente hasta la mirada multidimensional de Davis, en 1983, quien sustentó que la toma de perspectiva y la preocupación empática son fundamentales para entender este término.

Para diferenciar empatía de otros términos que se relacionan como simpatía y compasión, Schairer (2017) señaló que su uso indistinto es común por el parecido entre ellos; pero no significan lo mismo; por ejemplo, la simpatía es intentar entender lo que siente la otra persona; la empatía es sentir lo que otros sienten sea algo desagradable o feliz; en otras palabras, simpatizar es entender; mientras que empatizar es experimentar; en cambio, la compasión implica aliviar el dolor del otro e involucra necesariamente los dos términos anteriores.

Del mismo modo, Archer (2019) concluyó que la empatía es una cualidad social que se puede aprender y debe estar en el trabajo de cualquier profesional; sobre todo, de los médicos para bien de sus pacientes. Si no se separa las perspectivas del yo con el otro, solo se estaría simpatizando y no sería beneficioso para nadie porque produce agotamiento. Continuando la revisión del término, Bandrés (2018) indicó que las diferentes miradas disciplinarias coinciden en que la empatía es entender y participar en cómo se sienten las otras personas; por ende, carecer de esta capacidad, implicaría un problema social. El autor afirmó que las personas podemos empatizar ya que presentamos cerebros semejantes por las neuronas espejo descubiertas en 1996 que se activan al observar una conducta.

Por otro lado, Van Huyssteen (2017) precisó que la empatía es una de las tantas formas existentes para regular las emociones y las conductas de personas que presentan diferencias de toda índole. El teólogo explicó el papel relevante que juega la disposición empática como proceso sicológico en la evolución de la religión puesto que el cerebro humano está hecho para adaptarse a las creencias de una sociedad sean estas correctas o incorrectas, verdaderas o falsas. 
Ruiz (2018) mencionó que la forma de ser está marcada en los genes como es el temperamento; en cambio, el carácter se aprende. La autora indicó tres componentes de la personalidad: la instrospección, el neuroticismo y el psicoticismo. El neuroticismo tiene que ver con la regulación de las emociones que se encuentra asociado al sistema límbico. Mientras más alto se tenga el neuroticismo, las experiencias emocionales con las personas que te rodean son mucho más ricas.

Cherry (2019) enfatizó que lo esencial en la empatía es ponerse en el lugar de la otra persona; sobre todo, cuando esta sufre. La autora señaló que empatizar permite experimentar vivencias ajenas y sentirlas como propias además de que permite regular las propias emociones y tener conductas más adecuadas; no obstante, creer que las personas reciben lo que merecen o cosechan lo que siembran podría ser un factor que perjudique la capacidad de empatizar. Al respecto, Fragoso (2019) señaló que toda persona se encuentra en un proceso de socialización cuyo componente afectivo permite crear vínculos y salir exitoso de problemas cotidianos. La autora concluyó que la empatía es una competencia social que permite atender las emociones ya sean placenteras o no y experiencias ajenas de otras personas para, a partir de ello, regular las propias emociones.

En el campo de la docencia, Montoya (2018) reflexionó sobre la importancia de las emociones en la enseñanza humanística así como en la formación plena del alumnado. La autora afirmó que el docente juega un papel esencial en la regulación emocional de los educandos y entre sus competencias personales considera la asertividad, la claridad, el sentido del humor, el tacto, la diplomacia y, desde luego, la empatía. Siguiendo la línea de la empatía en las carreras pedagógicas, Gallardo (2018) señaló la importancia de implementar competencias emocionales en la malla curricular. El autor indicó que los maestros deben manejar conscientemente estas competencias; no obstante, deben ser capacitados en el manejo de estas herramientas desde su formación universitaria.

El autor base de esta investigación fue Davis (1980), quien luego de integrar muchos conceptos y estudios sobre el tema, define la empatía como las formas de reaccionar que tiene una persona ante las experiencias de otra. Creó un instrumento denominado Índice de Reactividad Interpersonal (IRI) que presenta un alto grado de confiabilidad. El IRI presenta 28 ítems con cinco alternativas repartidos en dos dimensiones de dos subescalas cada una. La dimensión cognitiva presenta las siguientes subescalas: 1) la toma de perspectiva que define como la tendencia a adoptar espontáneamente el punto sicológico de vista de otros, y 2) la fantasía que viene a ser cómo los encuestados pueden transponerse imaginativamente a los 
sentimientos y acciones de personajes ficticios de libros, películas y obras de teatro; mientras que la dimensión afectiva tiene las siguientes subescalas: 1) la preocupación empática que evalúa sentimientos de simpatía y preocupación orientados hacia otros desafortunados, y 2) la angustia personal que mide los sentimientos de ansiedad e incomodidad debido a las experiencias negativas de otras personas. La versión en español del IRI, realizada por tres traductores, fue la utilizada en esta investigación.

Cabe señalar que Davis (1996) indicó que la empatía es una forma más activa de entrar en la otra persona que el solo afecto compartido o simpatizar con alguien; es decir, empatizar implica comprender de una forma precisa lo que siente el otro y poder anticiparse a sus reacciones; o sea, mostrar agudeza social.

Reyes, Ruiz y Rodríguez (2018) desarrollaron una línea histórica sobre los conceptos de empatía a partir de la revisión de los trabajos investigativos de Davis. Los autores afirmaron que Davis presenta una definición que integra ambas dimensiones: la cognitiva y la emocional; entendiéndose la primera como una capacidad de sentir, comprender, ser consciente de pensamientos de las otras personas; mientras que la segunda se relaciona con la conducta o respuesta; es decir, hay un vínculo muy estrecho entre la empatía y el comportamiento de ayuda.

Seppälä, Simón, Brown, Worline, Cameron, Doty y Davis (2017) mostraron en un estudio que la empatía se evidencia en las relaciones interpersonales a través de la toma de perspectiva y de la compasión; siendo este último más débil que el primero pues la toma de perspectiva resultó ser más beneficiosa ya que implica intentar ponerse en los zapatos de otros antes de decidir o de criticar. Igualmente, Krogh, Medeiros, Bitran y Langer (2019) señalaron que las actitudes empáticas en el campo de la salud permiten mejor comprensión de la enfermedad; por consiguiente, son valoradas por los pacientes y las familias de estos.

Sobre la empatía, Goleman (2017) la definió como una competencia social que presenta dos dimensiones: la cognitiva y la emocional. La primera consiste en entender la perspectiva del otro; la segunda, en experimentar lo que la otra persona está sintiendo sin que te lo haga saber; o sea, captar señales. El autor también indicó que empatizar te convierte en un excelente líder y que se logra a través de una escucha profunda y una curiosidad que te permite relacionarte incluso con personas extrañas.

Más que una competencia social, Razzetti (2019) entendió la empatía como una superpotencia que se debe cultivar en tiempos en el que la era tecnológica torna a las personas a ser más aisladas. El autor señaló que la sensibilidad permite captar lo que otros sienten y 
que toda persona tiene la capacidad de empatizar si se lo propone, solo necesita de práctica y disciplina. En otro estudio, Razzetti (2019) confirmó que la tecnología nos desconecta con la sociedad y se observa la falta de empatía en estos tiempos sobre todo en estudiantes que se encuentran en formación profesional. Mientras que Bariso (2019) señaló que no es necesario que se haya pasado por las mismas circunstancias; pero sí que se haga algo al respecto; es decir, ayudar como se pueda (empatía compasiva); además mencionó que el solo hecho de intentar empatizar es una oportunidad de mejorar las relaciones sociales.

La empatía también influye en las relaciones económicas; al respecto, Garanti (2018) informó que promover la cultura de la empatía fue la clave del éxito para comprender las necesidades de los clientes de la entidad bancaria puesto que los usuarios sienten que se les escucha y que la institución financiera busca mejor su estilo de vida. Finalmente, Mandelli y Tonetto (2019) la definieron como un sentimiento que reduce la posibilidad de que surjan malos entendidos o problemas dentro del grupo social puesto que promueve relaciones saludables, enseña a tolerar las diferencias, alecciona a escuchar las opiniones contrarias y genera la preocupación por las necesidades de otros.

\section{Metodología}

La investigación presentó el enfoque cuantitativo que permitió recoger información y responder el problema de forma satisfactoria. Se aplicó el tipo básico sustantivo cuyo propósito es dar respuesta objetiva a un problema de la realidad social. (Carrasco, 2019). La investigación seleccionó el diseño no experimental descriptivo explicativo de predominancia puesto que busca observar y medir variables sin manipularlas intencionalmente dentro de una situación ya existente (Hernández y Mendoza, 2018, p. 174); por ello, la variable empatía no sufrió manipulación ni control alguno. La predominancia se refiere a una dimensión de la variable y luego a un indicador en específico de cada dimensión.

La población fueron estudiantes de Educación Primaria de dos universidades: la Universidad de Ciencias y Humanidades (UCH) y la Universidad Nacional Mayor de San Marcos (UNMSM); la muestra fue 139 alumnos de los últimos años de Educación Primaria: 86 estudiantes de la UCH y 53 de la UNMSM; esto implicó realizar el análisis de cada institución por separado.

La investigación necesitó una sola variable: empatía. Davis (1980) definió la empatía como las diversas maneras de reaccionar que una persona presenta ante las experiencias de 
otra. El sicólogo norteamericano mencionó que la empatía es una forma más activa y multidimensional de ingresar en las demás personas que simplemente compartir un afecto o simpatizar con alguien; es decir, empatizar implica comprender de una forma precisa lo que siente el otro y poder anticiparse a sus reacciones; o sea, mostrar agudeza social.

Se empleó la técnica de la encuesta. El instrumento que se empleó fue el Índice de Reactividad Interpersonal (IRI). En la ruta cuantitativa, el instrumento más empleado para el recojo de datos es un cuestionario que consiste en medir la variable a través de un conjunto de preguntas. Se utilizó el Test de Índice de Reactividad Interpersonal (IRI). El Cuestionario IRI consta de 28 ítems que se contestan en una escala de 5 puntos, que va desde poco a mucho. (Hernández y Mendoza, 2018). Para verificar si el IRI era confiable, se realizó una prueba piloto en 21 estudiantes. Se obtuvo un Alfa de Cronbach de 0,889 lo que indica que es confiable.

Antes de realizar el estudio, la autora se preguntó cómo afectará su investigación al proceso de enseñanza- aprendizaje. La investigación aportará a la comunidad académica puesto que la información recabada sobre empatía reducirá, de alguna manera, los problemas sociales en el mundo entero; además permitirá, tanto a las autoridades correspondientes como maestros de aulas, repotenciar las conductas empáticas en los estudiantes de Educación Primaria a partir de los resultados obtenidos. Luego se solicitó permiso a representantes de dos universidades peruanas: una pública y una privada, para poder realizar las encuestas a estudiantes de Educación Primaria. Las encuestas se aplicaron en un momento de la clase de los alumnos con previo permiso del maestro de turno y la disponibilidad de los participantes. Los datos de los participantes de ambas casas de estudio se mantuvieron en total reserva. La autora certifica que el trabajo es totalmente de su propiedad, que las fuentes utilizadas no fueron manipuladas ni los datos recabados como tampoco los resultados obtenidos.

\section{Resultados}

Luego de revisar antecedentes, un marco teórico sólido y aplicar el instrumento que se seleccionó para la recolección de datos, se obtuvieron resultados que fueron interpretados de acuerdo con las hipótesis y los objetivos planteados en esta investigación. El material gráfico elegido para presentarlos fue tablas.

Los resultados descriptivos e inferenciales que se alcanzaron luego de procesar la información obtenida del cuestionario IRI tomado a los 139 estudiantes de Educación 
Primaria tanto de la Universidad Nacional Mayor de San Marcos (UNMSM) como de la Universidad de Ciencias y Humanidades (UCH) son los que se detallan y explican a continuación a través de cinco tablas:

\section{Tabla 1.}

Frecuencias de la variable Empatía en estudiantes de Educación Primaria de dos universidades peruanas, 2019.

\begin{tabular}{ccc}
\hline Empatía & Frecuencia & Porcentaje \\
\hline Bajo & 24 & 17.3 \\
Moderado & 106 & 76.3 \\
Alto & 9 & 6.5 \\
Total & 139 & 100.0 \\
\hline
\end{tabular}

Se observó que la frecuencia que predominó en este resultado fue 'Moderado' con un 76.3\% (106 estudiantes), seguida del nivel 'Bajo' con un 17.3\% (24 personas). El nivel 'Alto' tuvo solamente un $6.5 \%$ del total de 139 evaluados.

\section{Tabla 2.}

Frecuencias de las dimensiones de la variable Empatía en estudiantes de Educación Primaria de dos universidades peruanas, 2019

\begin{tabular}{ccccccc} 
& $\mathrm{N}$ & $\%$ & $\mathrm{~N}$ & $\%$ & $\mathrm{~N}$ & $\%$ \\
\hline D1: Empatía Afectiva & 38 & 27.3 & 93 & 66.9 & 8 & 5.8 \\
D2: Empatía Cognitiva & 19 & 13.7 & 101 & 72.7 & 19 & 13.7
\end{tabular}

Se vio que ambas dimensiones la frecuencia que predominó fue la 'Moderado' con $66.9 \%$ y $72.7 \%$ para las dimensiones Empatía afectiva y Empatía Cognitiva respectivamente. Las siguientes frecuencias en ser las más elevadas estuvieron en la categoría 'Bajo' con $27.3 \%$ y $13.7 \%$ para las dimensiones 1 y 2 , respectivamente. 
Para los resultados inferenciales del objetivo general, se empleó la Regresión Logística Ordinal (RLO) con la finalidad de determinar cuál de las dimensiones fue la que más predominó en la variable Empatía. Para ello, se hicieron las estimaciones de parámetro teniendo en observación si fue o no significativo (valor p).

\section{Tabla 3.}

Resultados de las estimaciones de los parámetros mediante RLO de las dimensiones de la variable Empatía.

\begin{tabular}{|c|c|c|c|c|c|c|c|c|}
\hline & & $\begin{array}{l}\text { Estimación } \\
\iota\end{array}$ & $\begin{array}{l}\text { Desv. } \\
\text { Error }\end{array}$ & Wald & gl & Sig. & $\begin{array}{l}\text { Intervalo } \\
\text { al } 95 \% \\
\text { Límite } \\
\text { inferior }\end{array}$ & $\begin{array}{l}\text { e confianza } \\
\text { Límite } \\
\text { superior }\end{array}$ \\
\hline \multirow{2}{*}{ Umbral } & {$[$ Empatía = 1] } & $-36,114$ & 59,695 & ,366 & 1 &, 545 & $-153,115$ & 80,887 \\
\hline & [Empatía = 2] & $-8,119$ & 23,625 & , 118 & 1 & ,731 & $-54,423$ & 38,185 \\
\hline \multirow{6}{*}{ Ubicación } & $\begin{array}{l}\text { [D1_Empatía_Efectiv } \\
\mathrm{a}=1]\end{array}$ & $-20,161$ & 36,172 & ,311 & 1 & ,577 & $-91,057$ & 50,735 \\
\hline & $\begin{array}{l}\text { [D1_Empatía_Efectiv } \\
\mathrm{a}=2 \text { ] }\end{array}$ & $-9,323$ & 23,634 & , 156 & 1 & 693 & $-55,644$ & 36,998 \\
\hline & $\begin{array}{l}\text { [D1_Empatía_Efectiv } \\
\mathrm{a}=3]\end{array}$ & $0^{\mathrm{a}}$ & . & . & 0 & & . & . \\
\hline & $\begin{array}{l}\text { [D2_Empatía_cogniti } \\
\mathrm{va}=1]\end{array}$ & $-26,098$ & 43,505 & ,360 & 1 & ,549 & $-111,366$ & 59,169 \\
\hline & $\begin{array}{l}\text { [D2_Empatía_cogniti } \\
\mathrm{va}=2]\end{array}$ & $-15,191$ & 33,804 & ,202 & 1 & ,653 & $-81,446$ & 51,063 \\
\hline & $\begin{array}{l}\text { [D2_Empatía_cogniti } \\
\text { va=3] }\end{array}$ & $0^{\mathrm{a}}$ & . & . & 0 & & . & . \\
\hline
\end{tabular}

Función de enlace: Logit.

a. Este parámetro está establecido en cero porque es redundante.

Viendo las significancias, se indicó que todos los resultados parciales fueron superiores al nivel de significación ( $\mathrm{p}>0.05$ ), afirmando que ninguna dimensión fue predominante en la variable Empatía.

Para los resultados inferenciales del indicador predominante en la dimensión 1, se empleó la Regresión Logística Ordinal (RLO) con la finalidad de determinar cuál de los indicadores fue el que más predominó en la dimensión 1. Empatía Afectiva. Para ello se hicieron las estimaciones de parámetro teniendo en observación si fue o no significativo (valor $\mathrm{p}$ ). 


\section{Tabla 4.}

Resultados de las estimaciones de los parámetros mediante RLO de los indicadores de la dimensión Empatía afectiva.

\begin{tabular}{|c|c|c|c|c|c|c|c|c|}
\hline & & $\begin{array}{l}\text { Estimació } \\
\mathrm{n}\end{array}$ & $\begin{array}{l}\text { Desv. } \\
\text { Error }\end{array}$ & Wald & $\mathrm{gl}$ & Sig. & $\begin{array}{l}\text { Intervalo } \\
\text { al } 95 \% \\
\text { Límite } \\
\text { inferior }\end{array}$ & $\begin{array}{l}\text { e confianza } \\
\text { Límite } \\
\text { superior }\end{array}$ \\
\hline \multirow{2}{*}{ Umbral } & $\begin{array}{l}\text { [D1_Empatía Afectiva = } \\
\text { 1] }\end{array}$ & $-38,558$ & 155,300 & ,062 & 1 & ,804 & $-342,940$ & 265,824 \\
\hline & $\begin{array}{l}\text { [D1_Empatía Afectiva = } \\
\text { 2] }\end{array}$ & $-11,702$ & 70,126 & ,028 & 1 & ,867 & $-149,147$ & 125,743 \\
\hline \multirow{6}{*}{$\begin{array}{l}\text { Ubicaci } \\
\text { ón }\end{array}$} & [Indicador_PE=1] & $-23,678$ & 96,792 & ,060 & 1 & ,807 & $-213,386$ & 166,030 \\
\hline & [Indicador_PE=2] & $-11,009$ & 70,120 &, 025 & 1 & ,875 & $-148,441$ & 126,424 \\
\hline & [Indicador_PE=3] & $0^{\mathrm{a}}$ & . & . & 0 & . & . & . \\
\hline & [Indicador_AP=1] & $-26,702$ & 96,798 & ,076 & 1 & ,783 & $-216,422$ & 163,018 \\
\hline & [Indicador_AP=2] & $-13,494$ & 70,123 & ,037 & 1 & ,847 & $-150,932$ & 123,945 \\
\hline & [Indicador_AP=3] & $0^{\mathrm{a}}$ & . & . & 0 & . & . & \\
\hline
\end{tabular}

Función de enlace: Logit.

a. Este parámetro está establecido en cero porque es redundante.

Observando las significancias, se indica que todos los resultados parciales fueron superiores al nivel de significación ( $\mathrm{p}>0.05)$, afirmando que ningún indicador fue predominante en la dimensión Empatía afectiva.

Para los resultados inferenciales de la identificación del indicador predominante en la dimensión 2: Empatía Cognitiva, se empleó la Regresión Logística Ordinal (RLO) con la finalidad de determinar cuál de los indicadores fue el que más predominó en la dimensión 2. Empatía Cognitiva. Para ello se hicieron las estimaciones de parámetro teniendo en observación si fue o no significativo (valor p).

\section{Tabla 5.}

Resultados de las estimaciones de los parámetros mediante RLO de los indicadores de la dimensión Empatía cognitiva.

\begin{tabular}{ll|l|l|l|l|l|l|l}
\hline & & $\begin{array}{l}\text { Estimaci } \\
\text { ón }\end{array}$ & $\begin{array}{l}\text { Desv. } \\
\text { Error }\end{array}$ & $\begin{array}{l}\text { Wal } \\
\text { d }\end{array}$ & gl & Sig. & $\begin{array}{l}\text { Intervalo de confianza } \\
\text { al 95\% } \\
\text { Límite } \\
\text { inferior }\end{array}$ & $\begin{array}{l}\text { Límite } \\
\text { superior }\end{array}$ \\
\hline Umbral & $\begin{array}{l}\text { [D2_Empatía_cognitiva } \\
\text { 1] }\end{array}$ & $-39,513$ & 97,060 &, 166 & 1 &, 684 & $-229,747$ & 150,721 \\
\hline
\end{tabular}




\begin{tabular}{|c|c|c|c|c|c|c|c|c|}
\hline & $\begin{array}{l}\text { [D2_Empatía_cognitiva }= \\
2]\end{array}$ & $-11,081$ & 41,284 & ,072 & 1 & ,788 & $-91,996$ & 69,833 \\
\hline \multirow{6}{*}{$\begin{array}{l}\text { Ubicació } \\
\mathrm{n}\end{array}$} & [Indicador_TP=1] & $-20,346$ & 54,728 & , 138 & 1 &, 710 & $-127,610$ & 86,918 \\
\hline & [Indicador_TP=2] & $-11,487$ & 41,280 & 077 & 1 & ,781 & $-92,394$ & 69,421 \\
\hline & [Indicador_TP=3] & $0^{\mathrm{a}}$ & . & . & 0 & . & . & . \\
\hline & [Indicador_F=1] & $-28,537$ & 65,645 & , 189 & 1 & ,664 & $-157,199$ & 100,124 \\
\hline & [Indicador_F=2] & $-11,957$ & 41,283 & ,084 & 1 &, 772 & $-92,871$ & 68,957 \\
\hline & [Indicador_F=3] & $0^{\mathrm{a}}$ & . & . & 0 & . & . & . \\
\hline
\end{tabular}

Función de enlace: Logit.

a. Este parámetro está establecido en cero porque es redundante.

Viendo las significancias, se indicó que todos los resultados parciales fueron superiores al nivel de significación ( $p>0.05)$, afirmando que ningún indicador fue predominante en la dimensión Empatía cognitiva. Por último, se realizó el análisis estadístico por cada universidad puesto que una es pública y la otra, privada.

\section{Tabla 6.}

Frecuencias de la variable Empatía en estudiantes de la Universidad Nacional Mayor de San Marcos (pública)

\begin{tabular}{lcc}
\hline Válido & Frecuencia & Porcentaje \\
\hline Bajo & 2 & 3,6 \\
Moderado & 48 & 87,3 \\
Alto & 3 & 5,5 \\
Total & 53 & 100,0 \\
\hline
\end{tabular}

Se observó que la frecuencia que predominó en este resultado fue 'Moderado' con un 87.3\% (48 estudiantes), seguida del nivel 'Alto' con un 5.5\% (3 personas), por último, el nivel "Bajo" con un 3.6\% (2 alumnos).

\section{Tabla 7.}

Frecuencias de la variable Empatía en estudiantes de la Universidad de Ciencias y Humanidades (privada)

\begin{tabular}{lcc}
\hline Válido & Frecuencia & Porcentaje \\
\hline Bajo & 22 & 25,6 \\
Moderado & 56 & 65,1 \\
Alto & 8 & 9,3 \\
Total & 86 & 100,0
\end{tabular}


Se observó que la frecuencia que predominó en este resultado fue 'Moderado' con un $65.1 \%$ (56 estudiantes), seguida del nivel 'Bajo' con un 25.6\% (22 personas), por último, el nivel "Alto" con un 9.3\% (8 alumnos).

\section{Discusión y conclusión}

Se examinó y discutió el resultado alcanzado en la investigación con otros obtenidos en trabajos previos revisados, con la teoría desarrollada en el marco teórico y con las experiencias del día a día. De esta forma, se pudo explicar el resultado que se alcanzó a través de razonamientos válidos $\mathrm{y}$, finalmente, se logró aportes para próximos estudios que se quieran realizar utilizando la misma ruta.

Se juntaron las muestras de ambas universidades (UNMSM y UCH) en una sola sumando 139 en total de encuestados puesto que la intención no era compararlas ni encontrar en ellas coincidencias y discrepancias; sino describir y explicar la predominancia tanto de una dimensión como de un indicador de la variable empatía en estudiantes que se encuentran en los últimos ciclos de la carrera de Educación Primaria, que casi en su totalidad son mujeres y coinciden en la edad; sin embargo, los resultados indicaron que no hubo predominancia de ninguna dimensión ni de ningún indicador considerando que la investigación se realizó con toda la rigurosidad científica que exigía la investigación; es decir, se aplicó la regresión logística ordinal; no obstante, el estudio con los datos obtenidos no fueron evidencia suficiente para rechazar la hipótesis nula.

Al ser universidades de estratos distintos, se realizó un análisis estadístico de cada institución por separado y, coincidentemente, la frecuencia que destacó en ambas fue la moderada. Al respecto, Pulido y Herrera (2018) señalaron que las condiciones de vida influyen directamente en la forma de interactuar entre las personas; parece no ser el caso de estos centros de estudio cuyas frecuencias dominantes son similares; es decir, el aspecto socioeconómico no fue relevante; por otro lado, Archer (2019) señalaba que las actitudes empáticas se aprenden en cualquier espacio social independientemente de la cuestión económica; sin embargo, se esperaba que la universidad privada obtuviera un nivel alto predominante ya que los estudiantes pagan una pensión mensual.

Los trabajos previos, en su mayoría, se enfocaron al género de los participantes ya que estos eran estudiantes de Sicología y Medicina es, por ello, que se podía tener data 
homogénea de varones y mujeres; en el caso de esta investigación, no se podía realizar un estudio de género porque la presencia de varones que estudien la carrera de Educación Primaria es casi nula en Lima; no es el caso de España, como se observó en la investigación de Merino, Sánchez y Revilla (2018) que aplicaron el mismo cuestionario en 75 mujeres y 44 varones que estudian esta carrera. Lo anterior demostró que son realidades diferentes ya que los varones en nuestro país prefieren estudiar otras carreras alejadas a la educación, no es el caso en el extranjero. En el Perú, se observa la presencia mayoritaria de maestras en el nivel primario. La misma se va reduciendo en el nivel secundario en el que la presencia masculina se incrementa y así progresivamente. La Facultad de Educación de los niveles inicial y primario presenta más catedráticas que otras facultades.

Trabajos como el de Ulloque, Villalba, Varela, Fantini, Quinteros, Díaz (2019) y el de Fortich y Díaz (2018) fueron aplicados a estudiantes médicos de primero a quinto año de carrera; seguramente, por esa razón fue más simple encontrar diferencias empáticas entre ellos puesto que el nivel de involucramiento con la carrera es de manera progresiva y los que están por terminar empatizan un poco más; por tal razón, esta investigación rechazó la idea de realizar un estudio parecido.

Otra coincidencia en los trabajos revisados fue el empleo de muestras grandes como 811 participantes en el caso de Pulido y Herrera (2018), 693 participantes utilizaron Huarcaya y Encinas (2018), 450 participantes emplearon Giménez, Prado y Valero (2019) o 326 participantes como es el caso de Fortich y Díaz (2018). Los trabajos mencionados fueron los que presentaron las muestras más grandes lo que implicó una mayor financiación y tiempo de investigación; por lo anterior, sus estudios presentaron más objetivos, hipótesis, resultados y conclusiones. Un probable sesgo para esta investigación fue el no presentar una muestra más extensa que abarque más participantes de universidades distintas tanto de Lima como el interior del país; ello se debió a que se hubiese necesitado de más recursos económicos.

No se halló estudios parecidos exclusivos de empatía; sino asociados a otras variables como inteligencia emocional, comunicación, felicidad, entre otras. De igual modo, los estudios de empatía se han aplicado a estudiantes de colegio o a la empatía entre maestro y alumno como son las investigaciones de Maldonado y Barajas (2018) y Guzmán (2018); pero no se han aplicado a futuros profesores; o sea, antes de que dejen sus aulas universitarias y ejerzan su labor docente. Los estudios mencionados son de España y Costa Rica, respectivamente; es decir, otra prueba más de la ausencia de trabajos sobre empatía en el campo educativo a nivel local y nacional. 
En cuanto a trabajos iguales sobre predominancia de dimensión e indicador de la variable empatía, se halló solamente uno en el Perú; el de Corrales (2017). En el trabajo mencionado, se aplicó el mismo instrumento y sí se encontró predominancia; ello se debió, seguramente, a que el instrumento fue aplicado a estudiantes de Sicología; o sea, los alumnos de Educación Primaria llevan diversos cursos durante sus 5 años de formación profesional en los cuales aprenden a empatizar. Lo más probable es que los resultados obtenidos se deban a ello y que el nivel destacado haya sido el moderado; no obstante, esto no garantiza que, cuando ejerzan su labor, los 139 futuros maestros formen estudiantes de la mejor manera; esto es, haciendo uso de esta habilidad o competencia social, ello es lo esperado. A nivel internacional, no se halló trabajos.

Otro estudio parecido fue el de Mayo, Quijano, Ponce y Ticse (2019) que buscó diferencias significativas de empatía en residentes médicos por género y estado civil aplicando un instrumento diferente; pero no las encontró; no obstante, el nivel de empatía aumentó en todos aquellos que llevaron talleres, cursos o programas para mejorar sus relaciones interpersonales. Considerando que en esta investigación, los participantes serán futuros maestros que presenten un nivel moderado de empatía no parece ser lo más adecuado puesto que dentro de su plan curricular llevan cursos durante toda su formación universitaria.

Los resultados mostraron que los 139 participantes presentaron un nivel moderado de empatía debido a que llevan curso al respecto y ya Gallardo (2018) indicaba que los estudiantes de Educación, a diferencia de otros próximos profesionales, deben ser formados conscientemente con competencias sociales y emocionales durante sus años de preparación superior. Por todo lo anterior, se hubiera esperado que el nivel destacado de empatía fuese el alto.

El hecho de que no se haya encontrado trabajos sobre la empatía en estudiantes universitarios de Educación Primaria en el Perú y fuera del país demostró la importancia de trabajos como este y que sigan realizándose para medir el nivel de empatía en la que se encuentran los futuros maestros.

Hay que considerar que cada futuro docente formará entre 20 y 35 niños, si multiplicamos 139 futuros maestros por el número mínimo, el resultado sería 2780 niños que necesitan profesores bastante empáticos en tiempos que se evidencia mucha violencia e intolerancia en la realidad local, nacional e internacional tanto dentro de los hogares como fuera de ellos y se observa en las calles o, simplemente, a través de los medios de comunicación. 
A lo largo del tiempo, se ha buscado definir la variable empatía, llegando a una que integre ideas de muchos estudiosos. Se utilizó la definición del sicólogo norteamericano, Davis (1980), quien fue el autor base de esa investigación; por tal motivo se entendió la empatía de manera multidimensional abarcando no solo lo cognitivo; sino lo afectivo; es decir, saber cómo siente el otro o ponerse en su lugar; pero también compartir ese sentimiento y saber actuar al respecto. El fin de esta definición es poder anticiparse a posibles reacciones y evitar problemas: prevenir antes que lamentar. La definición de Davis es la más integradora como señalaron Reyes, Ruiz, Rodríguez y Palazón (2018).

En la investigación, no hubo resultados de predominancia de una dimensión sobre otra porque casi en la totalidad de participantes fueron mujeres. De 139 participantes 131 fueron señoritas que, además, no distaban en edades ni distancia entre ellas en tiempo de estudios porque se encontraban en los últimos ciclos de la carrera profesional.

Esta investigación lo que hizo fue ratificar las fuentes referenciadas en el campo de la pedagogía como el de Montoya (2018), quien señalaba que hay una relación estrecha entre ambas dimensiones de la empatía en un docente puesto que no solo es darse cuenta de las emociones del otro; sino, a partir de ellas, regular las propias como parte de la formación humanística integral de los futuros formadores. Davis (1980) señaló que la preocupación por cómo se está sintiendo el otro puede derivar a la conducta empática; esto es, hacer algo por la otra persona.

El hecho de que los participantes hayan elegido esta carrera ya muestra una disposición o ventaja para tener una mejor comunicación con el otro; o sea, el estudiante como señalaban Altuna (2018), Archer (2019) y Van Huyssteen (2017). Tanto hombres como mujeres tenemos la capacidad de empatizar según los estudios de neurociencias y las neuronas espejo que mencionaba Bandrés (2018). Otro posible sesgo en esta investigación fue el muestreo no probabilístico por conveniencia ya que este se emplea cuando se desconoce el número total de individuos que constituyen la población; por ende, no se puede generalizar los resultados obtenidos.

Sobre el tema de emociones, hay diferencias estructurales y funcionales; las primeras son indebatibles y tienen que ver con el sistema nervioso y los estudios neurológicos; en cuanto a las funcionales implica el cómo funciona la mente de los varones y de las mujeres de acuerdo al factor social; por ejemplo, algunos estudios dirán que el varón se desempeña mejor en matemáticas y las mujeres, en letras y viceversa; otros no estarán de acuerdo con estas afirmaciones; por ello, las diferencias funcionales sí son cuestionables; sin embargo, en 
nuestro contexto sociocultural, la misma sociedad va perfilando ciertas tendencias de personalidad; por ejemplo, la idea de que la mujer es más afectiva, soporta más las dificultades, los malos tratos, entre otros.

Sobre los tres componentes de la personalidad, Ruiz (2018) señalaba uno que era el neuroticismo; este factor mantenía en equilibrio las emociones; pero que el neuroticismo de las mujeres presentaba un nivel muy alto; por ende, la mujer presenta más riqueza emocional y empatizan más que los varones puesto que, como indicó la autora mencionada, ellos son emocionalmente más independientes. La autora dejó claro que los estudios al respecto no niegan que haya mujeres frías y hombres empáticos. Por todo lo anterior, las conclusiones de los resultados obtenidos fueron las siguientes:

Primera: No se determina significancia estadística para rechazar la hipótesis, afirmando que ninguna dimensión es predominante en la variable Empatía en los estudiantes de dos universidades peruanas. Es probable que la conclusión se deba a la relación muy estrecha entre ambas dimensiones (afectiva y cognitiva) como parte de la formación integral de cada estudiante; esta relación es más cercana en aquellas personas que se preparan para ser futuros maestros.

Segunda: No se determina significancia estadística para rechazar la hipótesis, afirmando que ningún indicador es predominante en la dimensión 1: Empatía Afectiva entre los estudiantes de dos universidades peruanas. La dimensión afectiva presenta los siguientes indicadores: 1) la preocupación empática que examina los sentimientos que se presentan hacia otros con menos fortuna, y 2) la angustia personal que calibra los sentimientos de ansiedad e incomodidad.

Tercera: No se determina significancia estadística para rechazar la hipótesis, afirmando que ningún indicador es predominante en la dimensión 2: Empatía Cognitiva entre los estudiantes de dos universidades peruanas. La dimensión cognitiva presenta las siguientes subescalas o indicadores: 1) la toma de perspectiva que se conceptualiza como la tendencia a adoptar espontáneamente el punto sicológico de vista de los demás, y 2) la fantasía que sería cómo los participantes que fueron encuestados pueden transponerse imaginativamente a los sentimientos y acciones de personajes no existentes. 


\section{Contribuciones de los autores:}

Ana María Carty-Vásquez, responsible de la concepción del estudio y revision final del artículo.

Janet Josco-Mendoza, elaboración de la metodología del artículo y revisión final.

Yolvi Ocaña-Fernández, elaboración de los resultados y discusión del artículo y revision final.

\section{REFERENCIAS}

1. Alves, D., Rodrigues, L. y Ribeiro, G. (2018). Altruism and empathy in situations involving unpredictable personal cost. Ciencias Psicológicas, 12(1), 7-15. https://dx.doi.org/10.22235/cp.v12i1.1589.

2. Altuna, B. (2018): "Empatía y moralidad: las dimensiones psicológicas y filosóficas de una relación compleja”, en Revista de Filosofía 43 (2), 245-262.

3. Arango, O., Olivera, A., Restrepo, V. y Puerta, I. (2018). Empathic skills and theory of mind in female adolescents with conduct disorder. Brazilian Journal of Psychiatry, 40(1), 78-82. Epub June 12, 2017.https://dx.doi.org/10.1590/1516-44462016-2092.

4. Archer, E, y Turner, R. (2019). Empathy: An essential tool in any doctor's skillset. SAMJ: South African Medical Journal, 109(1), 11-12. https://dx.doi.org/10.7196/samj.2019.v109i1.13732

5. Arenas, O., y Jaramillo, N. (2017). Concepciones de la empatía a nivel general, psicológico y a partir de sus instrumentos de medición. Colombia. Revista Psyconex, 9(15)2-8. Recogido https://aprendeenlinea.udea.edu.co/revistas/index.php/Psyconex/article/view/330991.

6. Bandrés, P. (2018).’La empatía desde la neurociencia". Estudio sobre la Empatía Profesional en estudiantes de medicina. Universidad Complutense de Madrid. España. 18- 25.

7. Bariso, J. (2019). There Are Actually 3 Types of Empathy. Here's How They Differ--and How You Can Develop Them All. The real world guide to emotional intelligence. EQ Applied. Recogido de https://www.inc.com/justin-bariso/there-are-actually-3typesofempathy-heres-how-they-differ-and-how-you-can-develop-them-all.html.

8. Bautista, G., Vera, J., Tánori, J., y Valdés, Á. (2016). Propiedades psicométricas de una escala para medir empatía en estudiantes de secundaria en México. Costa Rica. Revista Actualidades Investigativas en Educación, 16 (3), 1-20. Recogido de http://dx.doi.org/10.15517/aie.v16i3.25959.

9. Carrasco, S. (2019). Metodología de la Investigación Científica. Pautas metodológicas para diseñar y elaborar el proyecto de investigación. Editorial San Marcos. Decimonovena reimpresión.

10. Cherry, K. (2019). Importance and Benefits of Empathy. Recogido de https://www.verywellmind.com/what-is-empathy-2795562.

11. Concha, L., Ccarita, K., Marroquín, J., y Atamari, N. (2018). Evaluación de la empatía en estudiantes de medicina humana en una universidad pública de la sierra sur del Perú.

Revista Educación Médica, 327- 332, Recogido de https://doi.org/10.1016/j.edumed.2017.04.006.

12. Corrales, H. (2017). La empatía en estudiantes de psicología de dos universidades peruanas, 2017. Revista Docencia Universitaria, Recogido de http://repositorio.ucv.edu.pe/handle/UCV/14569. 
13. Dávila, Y., Neira, V., Aguilera, J., Martínez, F., Vélez, X., y Días, V. (2017). La empatía y estudiantes de medicina en la universidad de Azuay, Ecuador. Revista Científica Salud Uninorte, 33 (1), Recogido de http://dx.doi.org/10.14482/sun.33.1.10120.

14. Davis, M. (1980). A multidimensional approach to individual differences in empathy. JSAS Catalog of Selected Documents in Psychology, 10(4), 85-104. Fetzer Institute. 10, 85, 1-17.

15. Davis, M. (1996). Empathy. A Social Psychological Approach. JSAS Catalog of Selected Documents in Psychology.

16. Fortich, N., y Díaz, V. (2018). Actitud empática en estudiantes de Odontología de la Corporación Universitaria Rafael Núñez en la ciudad de Cartagena. Revista Ciencias $\begin{array}{lll}\text { de la Salud, 16(1), } & \text { 129-143. }\end{array}$ doi:http://dx.doi.org/10.12804/revistas.urosario.edu.co/revsalud/a.6495.

17. Fragoso, R. (2019). Importancia del desarrollo de la inteligencia emocional en la formación de personas investigadoras. Revista Actualidades Investigativas en Educación, 19 (1), DOI 10.15517/aie.v19i1.35410. Recogido de https://www.scielo.sa.cr/pdf/aie/v19n1/1409-4703-aie-19-01-655.pdf

18. Gallardo, A. (2018). Competencias emocionales y formación inicial docente en la Universidad de Playa Ancha. Revista Didac (72), 18- 23. Recuperado de http://revistas.ibero.mx/didac/uploads/volumenes/27/pdf/Didac72_web.pdf

19. Giménez, M., Prado, V. y Valero, S. (2019). Impact of work aspects on communication, emotional intelligence and empathy in nursing. Revista Latino-Americana de $\begin{array}{lllll}\text { Enfermagem, 27, e3118. Epub } & \text { February }\end{array}$ https://dx.doi.org/10.1590/15188345.2933.3118

20. Garanti, B. (2018). La cultura de la empatía, eje central del Informe Anual Integrado 2018 de Garanti. Recogido de https://www.bbva.com/es/la-cultura-de-laempatiaejecentral-del-informe-anual-integrado-2018-de-garanti/

21. Goleman, D. (2017). Empathy: A Key to Effective Leadership. Recogido de https://www.linkedin.com/pulse/empathy-key-effective-leadership-daniel-goleman

22. Guzmán, K. (2018). La comunicación empática desde la perspectiva de la educación inclusiva. Actualidades Investigativas en Educación, 18(3), 340358. https://dx.doi.org/10.15517/aie.v18i3.34211

23. Hernández- Sampieri, R., y Mendoza, C. (2018). Metodología de la Investigación. Las rutas cuantitativa, cualitativa y mixta. McGRAw- Hill interamericana editores, S.A

24. Huarcaya, J., y Encinas, M. (2018). Evaluación de la empatía en alumnos de Medicina humana de una universidad privada del Perú. Revista Educación Médica, 100- 107, Recogido de https://doi.org/10.1016/j.edumed.2018.05.008

25. Krogh, E., Medeiros, S., Bitran, M. y Langer, Á. (2019). Mindfulness y la relación clínica: pasos hacia una resiliencia en medicina. Revista médica de Chile, 147(5), 618627. https://dx.doi.org/10.4067/S0034-98872019000500618.

26. López, V., Arias, C., González, K., y García, K. (2018). Un estudio de la relación entre la empatía y la creatividad en alumnos de Colombia y sus implicaciones educativas. Revista Complutense De Educación, 29(4), 1133-1149. https://doi.org/10.5209/RCED.54881.

27. Maldonado, M. y Barajas, C. (2018). Teoría de la mente y empatía. Repercusiones en la aceptación por los iguales en niños y niñas de Educación Infantil, Primaria y Secundaria. Escritos de Psicología, 11, 10-24.

28. Mandelli, R. y Tonetto, L. (2019). Design para empatia: brinquedos e brincadeiras como oportunidade para promover o desenvolvimento emocional. Pesquisas e Práticas Psicossociais, 14(1), 1-22. Recuperado em 16 de outubro de 2019, de 
http://pepsic.bvsalud.org/scielo.php?script=sci_arttext\&pid=S1809890820190001000 $05 \& \operatorname{lng}=\mathrm{pt} \&$ tlng $=$.

29. Mayo, G., Quijano, E., Ponce, D. y Ticse, R. (2019). Utilización de la Escala de Empatía Médica de Jefferson en Residentes que realizan su especialización en un hospital peruano. Revista de Neuro-Psiquiatria. 82. 130. 10.20453/rnp.v82i2.3540.

30. Mendoza, J. (2018). Alfa de Cronbach-Psicometría con $R$. Recuperado de https://medium.com/@jboscomendoza/alfa-de-cronbach psicometr\%C3\%ADaconr55d3154806cf.

31. Merino, T., Sánchez, M., y Revilla, D. (2018). Perspectiva histórica y empatía: su interrelación en futuros profesores de Educación Primaria. España. Revista Aula Abierta, 47 (2), 221-228. Recogido de https://doi.org/10.17811/rifie.47.2.2018.221228.

32. Ministerio de Educación. (2017). La Ley General de la educación peruana. Recogido de http://www.minedu.gob.pe/comunicado/pdf/normativa-2018/ley-28044/ley28044leygeneral-de-educacion-24-11-2017.pdf

33. Montoya, L. (2018). La educación socioemocional en el contexto universitario. Revista Didac (72), 11-16. Recuperado de http://revistas.ibero.mx/didac/uploads/volumenes/27/pdf/Didac72_web.pdf

34. Moreira, L., Desouza, M. y Guerra, V. (2018). Self-Perception, Empathy and Moral SelfConcept Predict Moral Concerns in Adults. Paidéia (Ribeirão Preto), 28, e2818. Epub July 26, 2018.https://dx.doi.org/10.1590/1982-4327e2818.

35. Organización de las Naciones Unidas para la Educación, la Ciencia y la Cultura. (2017). Soporte socioemocional. Reconstruir sin ladrillos.

36. Organización de las Naciones Unidas para la Educación, la Ciencia y la Cultura. (2015). Educación para la ciudadanía mundial: Temas y objetivos de aprendizaje.

37. Palomino, P. y Almenara, C. (2019). Inteligencia Emocional en Estudiantes de Comunicación: Estudio Comparativo bajo el Modelo de Educación por Competencias. Revista Digital de Investigación en Docencia Universitaria, 13(1), 1- 16. https://dx.doi.org/10.19083/ridu.2019.840.

38. Pérez, G. (2019). Aportes desde la psicología educativa para el desarrollo de los valores y ética profesional. Revista de Investigacion Psicologica, (21), 11-37. Recuperado en 11 de octubre de 2019, http://www.scielo.org.bo/scielo.php?script=sci_arttext\&pid=S222330322019000100 $003 \& \operatorname{lng}=$ es\&tlng=es.

39. Pulido, F. y Herrera, F. (2018). Predictores de la felicidad y la inteligencia emocional en la educación secundaria. Revista Colombiana de Psicología, 27, 71-84. https://doi.org/10.15446/rcp.v27n1.62705.

40. Razzetti, G. (2019). Empathy Is the Superpower You Need. Recogido de https://www.psychologytoday.com/us/blog/the-adaptive-mind/201906/empathy- isthesuperpower-you-need.

41. Razzetti, G. (2019). Empathy is not a weakness but a superpower. Recogido de https://www.theladders.com/career-advice/empathy-is-not-a-weakness-butasuperpower.

42. Reyes, F., Ruiz, S. y Rodríguez, H. (2018). La adopción de perspectiva y la comunicación de la responsabilidad social corporativa: una revisión conceptual. Revista de la Facultad de Ciencias Económica: Investigación y Reflexión, XXVI (2), DOI: https://doi.org/10.18359/rfce.2888.

43. Ruiz, I. (2018). ¿Podemos cambiar de personalidad? El País. Recogido de https://elpais.com/elpais/2018/05/15/eps/1526380988_573301.html. 
44. Soto, R. (2015). La tesis de maestría y doctorado en 4 pasos. Biblioteca Nacional del Perú. Segunda edición.

45. Schairer, S. (2017). What's the Difference Between Empathy, Sympathy, and Compassion? Recogido de https://chopra.com/articles/whats-the-differencebetweenempathysympathy-and-compassion.

46. Seppälä, E., Simon, E., Brown, S., Worline, M., Cameron, D., Doty, J. y Davis, M. (2017). Empathy, Compassion, and Social Relationships. In The Oxford Handbook of Compassion Science. Oxford University Press. Retrieved 16 Oct. 2019, from https://www.oxfordhandbooks.com/view/10.1093/oxfordhb/9780190464684.001.000 1/oxfordhb-9780190464684-e-23.

47. Sevillano, S. (2016). El sistema de acreditación universitaria en el Perú: marco legal y experiencias recientes. Revista de Educación y Derecho. Education and Law Review número 15. Octubre 2016.

48. Sistema Nacional de Evaluación, Acreditación y Certificación de la Calidad Educativa. (2006). Recogido de https://www.peru.gob.pe/docs/PLANES/14243/PLAN_14243_2013_28740.pDF

49. Ulloque, M., Villalba, S., Varela, T., Fantini, A., Quinteros, S. y Díaz-Narváez, V. (2019). Empathy in medical students of Córdoba, Argentina. Archivos Argentinos de Pediatria, 117(2), 81-86. https://doi.org/10.5546/aap.2019.eng.81.

50. Van Huyssteen, W. (2017). Lecture three: From empathy to embodied faith: Interdisciplinary perspectives on the evolution of religion. HTS Theological Studies, 73(3), 1-11. https://dx.doi.org/10.4102/hts.v73i3.4488.

51. Zabala, M., Richard's, M., Breccia, F. y López, M. (2018). Relaciones entre empatía y teoría de la mente en niños y adolescentes. Pensamiento Psicológico, 16(2), 47-57. https://dx.doi.org/10.11144/javer;anacali.ppsi16-2.retm.

\section{SOBRE OS AUTORES:}

\section{Ana María Carty-Vásquez}

Universidad César Vallejo, Lima, Perú. E-mail: Chereana2006@ hotmail.com

(iD) http://orcid.org/0000-0003-4719-5223

\section{Janet Josco-Mendoza}

Universidad Continental, Lima, Perú. E-mail: jijosco@continental.edu.pe

(iD http://orcid.org/0000-0001-8544-269X

\section{Yolvi Ocaña-Fernández}

Universidad Privada San Juan Bautista, Lima, Perú. E-mail:

yolvi.ocana@upsjb.edu.pe

(iD) http://orcid.org/0000-0002-2566-6875

Recebido em: 24 de março de 2020

Aprovado em: 13 de maio de 2020

Publicado em: 01 de julho de 2020 